\title{
Anomalous behavior of the spin gap of a spin-1/2 two-leg antiferromagnetic ladder with Ising-like rung interactions
}

\author{
Takashi Tonegawa*, ${ }^{*}$, Kiyomi Okamoto², Shunsuke C. Furuya ${ }^{3}$, Tôru Sakai ${ }^{4,5}$ \\ ${ }^{1}$ Professor Emeritus, Kobe University, Kobe 657-8501, Japan \\ ${ }^{2}$ Department of Physics, Tokyo Institute of Technology, Tokyo 152-8551, Japan \\ ${ }^{3}$ DPMC-MaNEP, University of Geneva, 24 Quai Ernest-Ansermet CH-1211 Geneva, Switzerland \\ ${ }^{4}$ Graduate School of Material Science, University of Hyogo, Kamigori, Hyogo 678-1297, Japan \\ ${ }^{5}$ Japan Atomic Energy Agency, SPring-8, Sayo, Hyogo 679-5148, Japan
}

Received XXXX, revised XXXX, accepted XXXX

Published online XXXX

Key words: spin-1/2 two-leg ladder, anomalous behavior of the spin gap, degenerate perturbation theory, ground-state phase diagram, quantum phase transition, numerical diagonalization

*Corresponding author: e-mail tone0115@vivid.ocn.ne.jp

Using mainly numerical methods, we investigate the width of the spin gap of a spin-1/2 two-leg ladder described by $\mathcal{H}=J_{1} \sum_{j=1}^{N / 2}\left[\boldsymbol{S}_{j, a} \cdot \boldsymbol{S}_{j+1, a}+\boldsymbol{S}_{j, b}\right.$ $\left.S_{j+1, b}\right]+J_{\mathrm{r}} \sum_{j=1}^{N / 2}\left[\lambda\left(S_{j, a}^{x} S_{j, b}^{x}+S_{j, a}^{y} S_{j, b}^{y}\right)+S_{j, a}^{z} S_{j, b}^{z}\right]$, where $S_{j, a(b)}^{\alpha}$ denotes the $\alpha$-component of the spin-1/2 operator at the $j$-th site of the $a(b)$ chain. We mainly focus on the $J_{\mathrm{r}} \gg J_{1}>0$ and $|\lambda| \ll 1$ case. The width of the spin gap between the $M=0$ and $M=1$ subspaces ( $M$ is the total magnetization) as a function of $\lambda$ anomalously increases near $\lambda=0$; for instance, for $-0.1 \lesssim \lambda \lesssim 0.1$ when $J_{1} / J_{\mathrm{r}}=0.1$. The gap formation mechanism is thought to be different for the $\lambda<0$ and $\lambda>0$ cases. Since, in usual cases, the width of the gap becomes zero or small at the point where the gap formation mechanism changes, the above gap-increasing phenomenon in the present case is anomalous. We explain the origin of this anomalous phenomenon by use of the degenerate perturbation theory. We also draw the ground-state phase diagram.
1 Introduction Many papers on the spin ladder have been published so far [1]. Here we study the ground-state (GS) properties of an $S=1 / 2$ two-leg antiferromagnetic ladder with Ising-like rung interactions, which has not been investigated in detail until now. Our model is sketched in Fig 1 and expressed by

$$
\begin{aligned}
\mathcal{H}=J_{1} & \sum_{j=1}^{N / 2}\left[\boldsymbol{S}_{j, a} \cdot \boldsymbol{S}_{j+1, a}+\boldsymbol{S}_{j, b} \cdot \boldsymbol{S}_{j+1, b}\right] \\
& +J_{\mathrm{r}} \sum_{j=1}^{N / 2}\left[\lambda\left(S_{j, a}^{x} S_{j, b}^{x}+S_{j, a}^{y} S_{j, b}^{y}\right)+S_{j, a}^{z} S_{j, b}^{z}\right],
\end{aligned}
$$

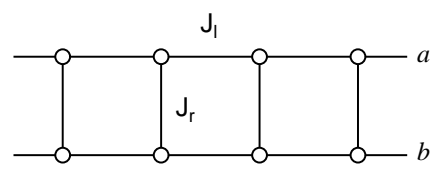

Figure 1 Sketch of the present model.

where $S_{j, a(b)}^{\alpha}$ denotes the $\alpha$-component ( $\left.\alpha=x, y, z\right)$ of the spin-1/2 operator at the $j$-th site of the $a(b)$ chain, and $N$ the number of spins which is supposed to be a multiple of 4 . The parameter $J_{1}$ denotes the magnitude of the leg interaction which is isotropic, and $J_{\mathrm{r}}$ denotes that of the rung interaction with the Ising-like anisotropy which is expressed by $\lambda$. We suppose $J_{1}>0$ and $J_{\mathrm{r}}>0$ and we 
restrict ourselves to the $J_{1} \ll J_{\mathrm{r}}$ and $|\lambda| \ll 1$ case. Hereafter we set $J_{\mathrm{r}}=1$ as the unit energy, In this paper we investigate the behavior of the spin gap and determine the GS phase diagram on the $\lambda-J_{1}$ plane mainly by use of the numerical methods.

2 Anomalous behavior of the spin gap Let us consider the spin gap defined by $\Delta_{01} \equiv E_{0}(M=$ $1, N)-E_{0}(M=0, N)$, where $E_{0}(M, N)$ is the lowest energy of the $N$ spin system in the subspace $M=\sum_{j}^{N / 2}\left(S_{j, a}^{z}+S_{j, b}^{z}\right)$. If $J_{l}=0$, the GS is the direct product of the rung state, where the rung state is either the singlet-dimer (SD) state $\frac{1}{\sqrt{2}}\left[\left(\begin{array}{l}\uparrow \\ \downarrow\end{array}\right)-\left(\begin{array}{l}\downarrow \\ \uparrow\end{array}\right)\right]$ or the triplet-dimer (TD) state $\frac{1}{\sqrt{2}}\left[\left(\begin{array}{l}\uparrow \\ \downarrow\end{array}\right)+\left(\begin{array}{l}\downarrow \\ \uparrow\end{array}\right)\right]$ depending on whether $\lambda>0$ or $\lambda<0$. The spin gap $\Delta_{01}$ is the energy difference between the SD state $(\lambda>0)$ or the $\operatorname{TD}(\lambda<0)$ state and the rung ferromagnetic state $\left(\begin{array}{c}\uparrow \\ \uparrow\end{array}\right)$, which is equal to $(1+|\lambda|) / 2$ having the minimum $1 / 2$ at $\lambda=0$. Thus, for sufficiently small leg interaction $J_{1} \ll 1$, it is expected that the spin gap $\Delta_{01}$ survives and its formation mechanism is different for the $\lambda>0$ and $\lambda<0$ cases. The change of the gap formation mechanism is often associated with the quantum phase transition (QPT). In usual QPT, we see three cases: (a) the spin gap $\Delta_{01}$ becomes small (but finite) at the first order QPT point, (b) becomes zero only at the second QPT point, (c) becomes zero in the finite range of the quantum parameter for the Berezinskii-Kosterlitz-Thouless QPT.

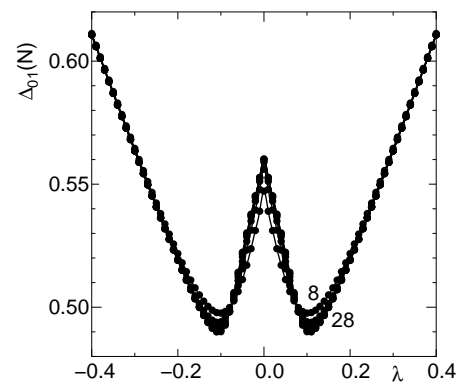

Figure 2 Numerical results of the spin gap $\Delta_{01}$ for $J_{1}=$ 0.1 with $N=8,12, \cdots, 28$. The $N$ dependence of $\Delta_{01}$ is reversed between the $\lambda \simeq 0.0$ case and the $\lambda \simeq 0.1$ case, although it is very weak.

Figure 2 shows the numerical results of the spin gap $\Delta_{01}$, calculated by the exact diagonalization (ED), for $J_{1}=$ 0.1 with $N=8,12, \cdots, 28$. As can be easily seen, the spin gap $\Delta_{01}$ always opens and clearly increases in the region $-0.1 \lesssim \lambda \lesssim 0.1$, which is quite different from the above expectations. Thus this behavior of the spin gap $\Delta_{01}$ is very anomalous. We note that the increase of $\Delta_{01}$ in both sides are due to the energy difference between the SD (or TD) and the ferromagnetic state, which is approximated by $1 / 2+|\lambda|$.
To explain the anomalous behavior of the spin gap $\Delta_{01}$, we use the degenerate perturbation theory considering the situation $J_{1} \ll 1$ and $|\lambda| \ll 1$. First we consider the rung state with $J_{1}=0$, which is nothing but the twospin problem. The eigenstates are the ferromagnetic states $\left(\begin{array}{l}\uparrow \\ \uparrow\end{array}\right)$ and $\left(\begin{array}{l}\downarrow \\ \downarrow\end{array}\right)$ with the energy $1 / 4$, the SD state with the energy $-(1+2 \lambda) / 4$, and the TD state with the energy $-(1-2 \lambda) / 4$. Here we take the SD and TD states into consideration, neglecting two ferromagnetic states with higher energies. We introduce the pseudo-spin $\boldsymbol{T}$ to express these two states. Namely, the eigenstates of $T^{z}$ with the eigenvalues $+1 / 2$ and $-1 / 2,|\Uparrow\rangle$ and $|\Downarrow\rangle$, correspond to the SD state and the TD state, respectively. Next, by taking the effect of $J_{1}$ into account in the lowest order we obtain the effective Hamiltonian

$$
\mathcal{H}_{\text {eff }}=2 J_{1} \sum_{j=1}^{N / 2} T_{j}^{x} T_{j+1}^{x}-\lambda \sum_{j=1}^{N / 2} T_{j}^{z}-\frac{N}{8} .
$$

Since both of $|\Uparrow\rangle$ and $|\Downarrow\rangle$ have zero magnetization in the original spin picture, $\mathcal{H}_{\text {eff }}$ describes only the $M=0$ subspace. The 'magnetic field term' (the second term in the rhs of eq.(2) comes from the energy difference between the $|\Uparrow\rangle$ and $|\Downarrow\rangle$ states. The effective Hamiltonian $\mathcal{H}_{\text {eff }}$ is essentially the one-dimensional transverse field Ising model exactly solved by Pfeuty [2]. The GS of $\mathcal{H}_{\text {eff }}$ is either the disordered state or the Néel ordered state in the $x$-direction ( $x$-Néel state) according as $|\lambda|>J_{1}$ or $|\lambda|<J_{1}$. The QPT between these two states is of the two-dimensional Ising type. In the disordered phase only the expectation value $\left\langle T_{j}^{z}\right\rangle$ is nonzero; $\left\langle T_{j}^{z}\right\rangle>0$ for $\lambda>0$ and $\left\langle T_{j}^{z}\right\rangle<0$ for $\lambda<0$. Thus, the rungs are essentially in the SD state for $\lambda>J_{1}$ and in the TD state for $\lambda<-J_{1}$. For the $x$-Néel phase, the eigenstates of $T_{j}^{x}$ are $\frac{1}{\sqrt{2}}(|\Uparrow\rangle+|\Downarrow\rangle)=\left(\begin{array}{l}\uparrow \\ \downarrow\end{array}\right)$ and $\frac{1}{\sqrt{2}}(-|\Uparrow\rangle+|\Downarrow\rangle)=\left(\begin{array}{l}\downarrow \\ \uparrow\end{array}\right)$ with the eigenvalues $+1 / 2$ and $-1 / 2$, respectively. Thus the $x$-Néel state in the $T$ picture is the $z$-Néel state of the original $S$ picture. The Néel state is realized by the cooperative effect between the SD and TD states induced by $J_{1}$. Three GSs are sketched in Fig 3 . Figure 4 shows the GS phase diagram based on $\mathcal{H}_{\text {eff }}$.

The physical interpretation of the above calculation is as follows. The energy difference between the SD and TD states per rung is $|\lambda|$. When the perturbation energy $J_{1}$ is smaller than this energy difference, $J_{1}<|\lambda|$, either the SD state or the TD state becomes important for the rung state. On the other hand, when the perturbation energy $J_{1}$ is larger than this energy difference, $J_{1}>|\lambda|$, both of the SD and TD states are cooperatively concerned with the formation of the rung state. The linear combination of these two state with equal weight brings about the rung Néel state.

The Néel state is a doubly degenerate state, while the SD and TD states are unique and protected by symmetry. Namely, if we exchange the upper and lower legs, the wave function of the SD state changes its sign and that of the TD state is unchanged. For the Néel state, this operation will change a wave function to the other wave function of 

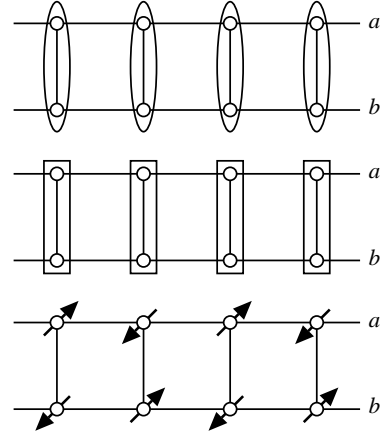

Figure 3 Sketches of the three GSs, i.e., the SD, TD, and Néel states in the original $S$ picture, from top to bottom. Ellipses show the SD pairs and rectangles the TD pairs.

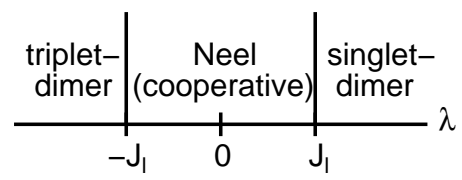

Figure $4 \mathrm{GS}$ of the present model by use of $\mathcal{H}_{\mathrm{eff}}$.

the doubly degenerate GS. The GSs of finite systems are usually unique and do not exhibit the spontaneous symmetry breaking. For the Néel state, a low-lying excited state with $M=0$ in the finite system asymptotically degenerate to the GS as $N \rightarrow \infty$, and the reconstruction of these two states by the linear combination results in the doubly degenerate Néel state with spontaneous symmetry breaking. Thus, in the Néel region, the lowest excitation energy within the $M=0$ subspace, $\Delta_{00}^{(1)} \equiv E_{1}(M=$ $0)-E_{0}(M=0)$, will rapidly decrease with the increase of the system size $N$. Here $E_{1}(M=0)$ is the energy of the first excited state within the $M=0$ subspace. The left panel of Fig 5 shows the numerical results of $\Delta_{00}^{(1)}$ for $J_{1}=0.1$ by the ED, in which we can clearly see the abovementioned behavior for $-0.1 \lesssim \lambda \lesssim 0.1$. The true gap within the $M=0$ subspace is $\Delta_{00}^{(1)}$ in the SD and TD regions, while it is $\Delta_{00}^{(2)} \equiv E_{2}(M=0)-E_{0}(M=0)$ in the Néel region, where $E_{2}(M=0)$ is the energy of the second excited state within the $M=0$ subspace. The behavior of $\Delta_{00}^{(2)}$ is shown in the right panel of Fig.5.

Let us define the shift of the GS energy due to $J_{1}$ by

$$
\Delta E_{0}(M=0) \equiv E_{0}\left(M=0, J_{1}\right)-E_{0}\left(M=0, J_{1}=0\right) \text {. }
$$

If we use $\mathcal{H}_{\text {eff }}$, we can obtain

$$
\begin{aligned}
& \Delta E_{0}(M=0) \\
& =-\frac{N}{2}\left\{\frac{1}{\pi} \int_{0}^{\pi / 2} \sqrt{J_{l}^{2}+2 J_{1} \lambda \cos k+\lambda^{2}} d k-\frac{\lambda}{2}\right\}
\end{aligned}
$$

Figure6 shows the behavior of $\Delta E_{0}(M=0)$ for $J_{1}=0.1$.
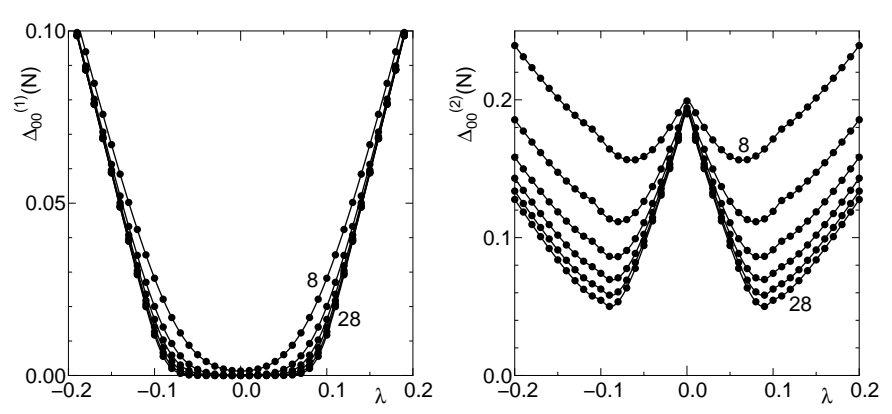

Figure 5 Behaviors of $\Delta_{00}^{(1)}$ (left) and $\Delta_{00}^{(2)}$ (right) for the $J_{1}=0.1$ case. The quantity $\Delta_{00}^{(2)}$ closes in the $N \rightarrow \infty$ limit at the QTPs within the numerical accuracy.

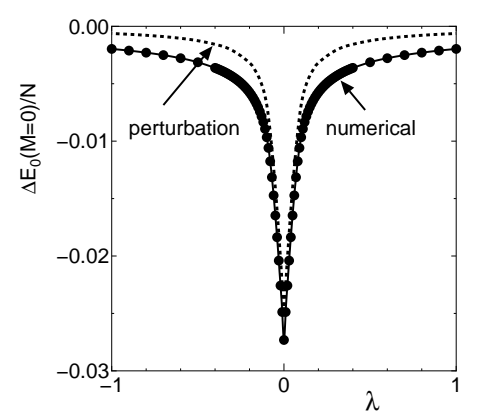

Figure 6 Shift of the GS energy $\Delta E_{0}(M=0)$ for the $J_{1}=0.1$ case. Dots denote the numerical results with $N=$ 28 and broken lines the results of the perturbation theory eq. (4).

As can be seen in Fig. 6, the decrease of the GS energy due to $J_{l}$ is much larger in the Néel region than in the SD and TD regions. The $M=1$ lowest state can essentially obtained from the GS by changing a rung pair (Néel pair, SD pair or TD pair) to the ferromagnetic pair, although the location of the ferromagnetic pair is not fixed. Thus, this energy cost, which is essentially the spin gap $\Delta_{01}$, is larger in the Néel region than in the SD and TD regions. This is a physical explanation for the anomalous behavior of the spin gap $\Delta_{01}$ shown in Fig. 2] We note that we cannot directly calculate the spin gap $\Delta_{01}$ by use of the effective Hamiltonian $\mathcal{H}_{\text {eff }}$, because it describes only the $M=0$ subspace.

3 GS phase diagram The boundary between the Néel and SD (or TD) phases can be numerically determined by use of the phenomenological renormalization group (PRG) method [3]. Namely, the critical value $\lambda_{\mathrm{c}}$ for fixed $J_{1}$ can be obtained by $\lambda_{\mathrm{c}}=\lim _{N \rightarrow \infty} \lambda_{\mathrm{c}}(N, N+$ $4)$, where $\lambda_{\mathrm{c}}(N, N+4)$ is the numerical solution of $N \Delta_{00}^{(1)}(N, \lambda)=(N+4) \Delta_{00}^{(1)}(N+4, \lambda)$. Figure 7 shows the phase diagram of the present model on the $\lambda-J_{1}$ plane. The spin gaps $\Delta_{00}^{(1)}$ and $\Delta_{00}^{(2)}$ within the $M=0$ subspace 


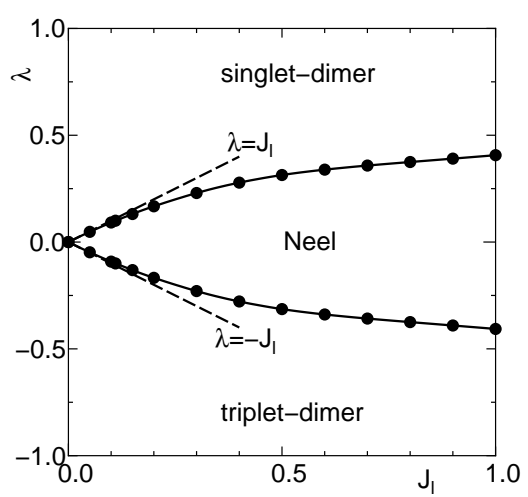

Figure 7 GS phase diagram of the present model determined by the ED with the PRG method. Dots denote the numerical results and broken lines the results of the perturbation theory $\lambda=J_{1}$.

close on the phase transition lines, whereas the spin gap $\Delta_{01}$ always opens. Thus, on the phase transition lines, at sufficiently low temperatures, the specific heat $C(T)$ shows the gapless behavior, while the magnetic susceptibility $\chi_{z}(T)$ for the magnetic field along the $z$ direction shows a gapful behavior. In usual cases, both of the spin gaps within the $M=0$ subspace and that between the $M=0$ and $M=1$ subspaces close simultaneously on the phase transition line, which leads to the gapless behaviors of both of $C(T)$ and $\chi_{z}(T)$ at low temperatures.

4 Discussion and concluding remarks Let us compare the present model with the $S=1 / 2$ bondalternating Heisenberg chain

$$
\mathcal{H}_{\text {ba }}=\sum_{i=1}^{N}\left[1+(-1)^{j} \delta\right] \boldsymbol{S}_{j, a} \cdot \boldsymbol{S}_{j+1, a} .
$$

where $\delta(-1 \leq \delta \leq 1)$ denotes the bond-alternation parameter and $N$ the number of spins which is supposed to be even. This bond-alternation model is a typical model which exhibits the change of the gap formation mechanism. When $\delta \neq 0$, the spin gap $\Delta_{01}$ is finite even in the thermodynamic limit as $\Delta_{01} \sim|\delta|^{2 / 3} / \sqrt{|\log | \delta||}$ [4,5]. The SD pairs exist on the $(1+\delta)$-bonds for $\delta>0$ whereas they exist on the $(1-\delta)$-bonds for $\delta<0$. When $\delta=0$, the GS is the Tomonaga-Luttinger (TL) liquid state with the gapless excitation. Thus the second-order QPT occurs at $\delta=0$. This situation is sketched in Fig. 8 As can be seen from Fig. 8. the location of the SD pair is different for the $\delta>0$ and $\delta<0$ cases. In other words, the unit cell which is important for the gap formation is reconstructed when $\delta$ pass through the QPT point $\delta=0$. On the other hand, for the present model case, the unit cell is always a rung and the reconstruction of the unit cell does not occur, as is shown in Fig. 3. Only the change of the GS of the unit cell occurs when $\lambda$ is swept. This is the very important and essential point for the quite different behavior of the spin gap $\Delta_{01}$ of the present model and the $S=1 / 2$ bond-alternating Heisenberg chain model.

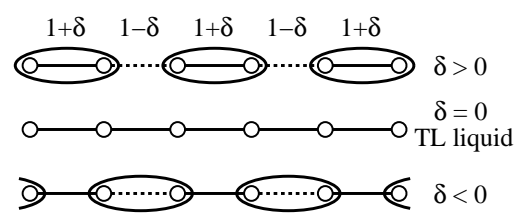

Figure $8 \mathrm{GSs}$ of the $S=1 / 2$ bond-alternating Heisenberg chain $\mathcal{H}_{\text {ba }}$. Ellipses denote the SD pairs. The locations of ellipses are different for the $\delta>0$ and $\delta<0$ cases.

As stated above, the key points to the anomalous behavior of the spin gap $\Delta_{01}$ are "no reconstruction of the unit cell" and "change of the GS of the unit cell". Thus, in other models with these properties, similar behavior of the spin gap $\Delta_{01}$ is expected. In fact, in the $S=1 / 2$ three-leg isosceles spin nanotube, a similar behavior of the width of the magnetization plateau at $1 / 3$ of the saturation magnetization is observed [6].

The anomalous behavior of the spin gap $\Delta_{01}$ seems to remain even for the the $J_{1} \gg 1$ and $0>J_{1} \gg-1$ cases. We have also succeeded in explaining this anomalous behavior for the former case by use of the bosonization approach. The details will be published elsewhere.

Finally we note that a closely related model was investigate by Zheng-Xin Liu et al. [7] from a somewhat different point of view.

In conclusion, by numerical methods, we have observed an anomalous behavior of the spin gap $\Delta_{01}$ of the spin-1/2 two-leg antiferromagnetic ladder with Ising-like rung interactions. Also we have succeeded in explaining the physical origin of this anomalous behavior.

Acknowledgements This work was partly supported by grants-in-aid for Scientific Research (B) (no. 23340109) and Scientific Research (C) (no. 23540388), from the Ministry of Education, Culture, Sports, Science, and Technology of Japan. S.C.F. acknowledges Institute for the Solid State Physics, the University of Tokyo, where a part of this work was carried out. We thank the Supercomputer Center, the Institute for Solid State Physics, the University of Tokyo, for computational facilities.

\section{References}

[1] For instance, Y. Nishiyama, N. Hatano and M. Suzuki, J. Phys. Soc. Jpn. 641967 (1995); E. Daggotto and T. M. Rice, Science 271618 (1996); G. I. Japaridze, A. Langari and S. Mahdavifar, J. Phys.: Condens. Matter 19076201 (2007).

[2] P. Pfeuty, Ann. Phys. (N. Y.) 5779 (1970).

[3] M. P. Nightingale, Physica A 83561 (1976).

[4] M. C. Cross and D. S. Fisher, Phys. Rev. B 19402 (1979).

[5] J. L. Black and V. J. Emery, Phys. Rev. B 23429 (1981).

[6] T. Sakai, M. Sato, K. Okamoto, K. Okunishi and C. Itoi, J. Phys.: Condens. Matter 22403201 (2010).

[7] Zheng-Xin Liu, Zhen-Biao Yang, Yong-Jian Han, Wei Yi and Xiao-Gang Wen, arXiv:1204.5162 